\title{
Forget Me Not: Mental Health Services for Older People
}

\author{
Audit Commission Report January 2000
}

The exclusion of services for older people from the Mental Health National Service Framework and the delays in publication of the National Service Framework for the elderly have caused many old age psychiatrists to be concerned that, as in many other areas of health service planning and provision, the needs of older people are not being adequately addressed by Government and health service planners. The recent Audit Commission Report, entitled Forget Me Not: Mental Health Services for Older People ${ }^{\dagger}$, gives us much encouragement that the needs of older adults have not been forgotten. Its recent publication should provide an authoritative focus, which will help old age psychiatrists, their multi-disciplinary and management colleagues and commissioners of services to push forward improvements in services for older people with mental illnesses.

The Audit Commission has been quietly reviewing public sector services for older people with mental health problems over the past 18 months and this is the first report in a series. It considers the range of specialist services to be provided; examines relationships with primary care and the residential and nursing home sector; and addresses issues concerned with the coordination of services including new powers under the National Health Service Act 1999, which allow pooled funding, a means of encouraging integrated provision and much greater flexibility when providing mental health services for older adults.

\section{Old age psychiatry services}

The main emphasis is on dementia, including care of people with dementia aged below 65 years. This might be an opportunity missed, but depression and other mental illnesses are included and the report states that better treatment of older people with depression "could result in cost savings, as people who recover from depression tend to use fewer services".

The report emphasises the need for comprehensive assessment and encourages home assessments. Caseloads and dependency levels of patients within the service should be monitored and the skill mix of staff should be reviewed and rebalanced if necessary. Information and advice should be readily available for carers and might include lectures, training programmes and information in various languages.

Out-patient and day services are exhorted to offer a range of therapies. Day hospitals are to be used for time-limited assessment and treatment. Hospital provision should be available for respite care, plus respite in residential/nursing care and home-based respite. The need for separate day hospital and in-patient areas for people suffering from a dementia and those with functional illnesses is pointed out. A person with designated responsibility for early-onset dementia is recommended in all areas.

\section{Relationship with primary care}

General practitioners (GPs) and primary care teams are seen as crucially important, but often in need of more support and advice from specialist mental health professionals. The report recommends that low-referring GPs should be contacted and offered support and training. GPs' views should be sought. Referral protocols should be developed to help GPs identify memory problems, depression or other signs of mental illness in late life. The use of standardised assessment tests (eg. Mini-Mental State Examination (Folstein et al, 1975), Geriatric Depression Scale (Yesavage, 1988)) by GPs should be encouraged. GPs should refer users and carers to local support groups at an early stage.

\section{Residential/nursing homes}

Health and social services should work together to provide good-quality care in residential and nursing homes. There should be a willingness to fund places in specialist residential care for people with dementia at a higher rate to reflect the need for specialist skills in their care. Concern is evident regarding variations in the use of antipsychotic medication in homes. Doses of medication should be as low as possible and reviewed regularly. An important quality indicator is the number of moves of placement. NHS bodies should not relinquish responsibility for continuing care (a particularly welcome assertion) and health and social services should have agreed criteria for eligibility for continuing NHS care.

\section{Coordination of services}

Effective communication, shared expertise and the need to exchange information are stressed. This might include joint assessments/reviews, use of the Care Programme Approach, shared management plans and specialist home-care workers. A local comprehensive strategy for these services is needed. This should be developed with users, carers, professionals and independent providers, and it should examine process and outcome indicators. Services should be well coordinated and jointly managed services should be considered. $\dagger$ Forget Me Not: Mental Health Services for Older People is published by the Audit Commission and is available from Audit Commission Publications, Bookpoint Limited, 39 Milton Park, Abingdon,

Oxon OX14 4TD (tel. 0800 502030) at $f 20$ 
可

editorials

\section{Conclusion}

The Audit Commission Report is a helpful and sensible document, which can give an impetus to the development of mental health services for older adults. It will complement the work of the National Service Framework for the elderly, which is eagerly awaited. We advise all those working in old age psychiatry, and those who commission services, to familiarise themselves with this work. We also commend the document to specialists in other areas of psychiatry, particularly where there is interest in coordination of services and working with primary care.

\section{References}

FOLSTEIN, M. F., FOLSTEIN, S. \&

YESAVAGE, G. (1988) Geriatric MCHUGH, P. R. (1975) Mini-mental state: a practical method for grading the cognitive state of patients for the Depression Scale.

clinician. Journal of Psychiatric Research, 12, 189-198.

Susan M. Benbow Consultant Psychiatrist (Old Age Psychiatry), Carisbrooke Resource Centre, Wenlock Way, Gorton, Manchester M12 5LF,

Sean P. Lennon Consultant Old Age Psychiatrist, Withington Hospital, Manchester 